\title{
Application of Markov Process in Performance Analysis of Feeding System of Sugar Industry
}

\author{
S. P. Sharma and Yashi Vishwakarma \\ Department of Mathematics, Indian Institute of Technology Roorkee, Roorkee-247667, Uttarakhand, India \\ Correspondence should be addressed to Yashi Vishwakarma; yashidec23@gmail.com
}

Received 24 January 2014; Accepted 30 March 2014; Published 27 April 2014

Academic Editor: Qiguang Miao

Copyright (c) 2014 S. P. Sharma and Y. Vishwakarma. This is an open access article distributed under the Creative Commons Attribution License, which permits unrestricted use, distribution, and reproduction in any medium, provided the original work is properly cited.

\begin{abstract}
To analyse the performance measures of complex repairable systems having more than two states, that is, working, reduced and failed, it is essential to model suitably their states so that the system governs a stochastic process. In this paper, the application of time-homogeneous Markov process is used to express reliability and availability of feeding system of sugar industry involving reduced states and it is found to be a powerful method that is totally based on modelling and numerical analysis. The selection of appropriate units/components in designing a system with different characteristics is necessary for the system analyst to maintain the failure-free operation. Keeping this concept in this study, the steady state availability of concern system is analysed and optimized by using a popular search technique, genetic algorithm. The objective of this paper is to consider the system operative process as Markov process and find its reliability function and steady state availability in a very effective manner and also to obtain an optimal system designing constituents which will allow a failure-free operation for long time period as required for maximum system productivity. The system performance measures and optimized design parameters are described and obtained here by considering an illustrative example.
\end{abstract}

\section{Introduction}

Process industries like chemical industry, sugar mill, thermal power plant, oil refineries, paper plant, fertilizer industry, and so forth have major importance in real life situations as they fulfil our various unavoidable requirements. The demand of product quality and system reliability is on an increase day by day. Further the failure is a random phenomenon, always associated with the operating state of any physical system and its causes are either deterioration in the components of the system and/or man handling errors. Therefore the main concern is to maintain system performance measures such as reliability and availability to achieve high profit goals and productivity in regard to system failures. These measures are considered as most significant factors associated with nonrepairable and repairable systems, respectively [1]. Nowadays industries are becoming quite complex in structure due to the advancement in the technology and therefore it is a difficult job for plant personnel/system analyst to decide proper maintenance policy. By properly designing the system, the factors such as performance, quality, productivity and profit, can easily be enhanced up to the desired goal of demand [2]. While designing, it is convenient to demonstrate the system behaviour among its units having varying failure and repair policies. Hence the job of plant personnel or system analyst is to examine, specify, and determine the system behaviour so that the acceptable choice of components will assist in increasing the efficiency of the system.

In the literature various techniques have been used to analyze the behaviour of systems and find their reliability, availability, and maintainability. Some widely used techniques are event tree, fault tree analysis (FTA), reliability block diagrams (RBD), Petri nets (PN), and Markovian approach, and so forth which perform by modelling the states of the system or the system [3-7]. Dhillion and Singh [8] have taken many systems to demonstrate their behaviour by using various engineering techniques. Sharma and Garg [9] and Garg and Sharma [10] have modelled the system failure event by Petri nets and then analysed the behaviour of urea decomposition system and synthesis unit in fertilizer 
plant, respectively. Kumar et al. [11, 12] have analysed the reliability and availability behaviour of subsystems of paper industry and sugar industry by modelling their states and using probabilistic approach. The availability analysis of steam and power generation systems in thermal power plant has been done by Arora and Kumar [13] by using ChapmanKolmogorov birth-death process. Gupta [14] has analysed the operational behaviour of a chemical production system by using a methodology based on Markov modelling. Kumar and Tewari [15] have modelled the $\mathrm{CO}_{2}$ cooling system of a fertilizer plant by Markov birth-death process and then optimized the system performance by genetic algorithm. Gupta et al. [16] have dealt with the system reliability and availability in butter oil processing plant by using Markov process and Runge-kutta method. Kumar et al. [17] have proposed a method based on Markov approach to evaluate an availability simulation model in a thermal power plant. Recently Manglik and Ram [18] have analysed the reliability of a two-unit cold standby system using Markov process and Kumar [19] has done the availability analysis of thermal power plant boiler air circulation system by using Markov approach. Therefore, it is found from the literature that the Markov approach has become a popular technique among other methods for the behavioural analysis and modelling of systems. In order to enhance the performance measures by selecting suitable choices of units, it is necessary to consider an efficient optimization technique. There is a variety of methods available in the literature [20-22]. The reliability of industrial systems using soft-computing based technique has been analysed by Garg et al. [23]. One of the optimization techniques, genetic algorithm (GA) which resembles the phenomenon of natural selection and reproduction, is a random search technique and it has been used for various reliability optimization problems. Khanduja et al. [24] have used GA with Markovian approach for paper making system of paper industry. The series parallel system was studied by Sharma et al. [25] and best design policy is obtained using GA. Thus GA is found to be a powerful technique as it provides a robust search and is not limited to the restricted search spaces.

In the present paper the importance of Markov process is shown by analysing the reliability function and availability of feeding system of sugar industry. The Markov transition diagram indicating the system states is presented as it gives availability function and the updated diagram leads to the expression for reliability function. While applying the normalizing condition, the expression for steady state availability is obtained and optimized by using genetic algorithm. Software package MATLAB with genetic algorithm toolbox is used for the execution of GA to the optimization problem. Thus the optimum design parameters obtained can give system analyst the best policy for high availability and productivity of the system. The purpose of this study is to obtain (1) reliability function and availability function both from Markov diagram of feeding system of sugar industry, (2) an expression of steady state availability optimization model with components' constant failure and repair rates, and (3) optimal system parameters by employing GA and a decision of system design policy.

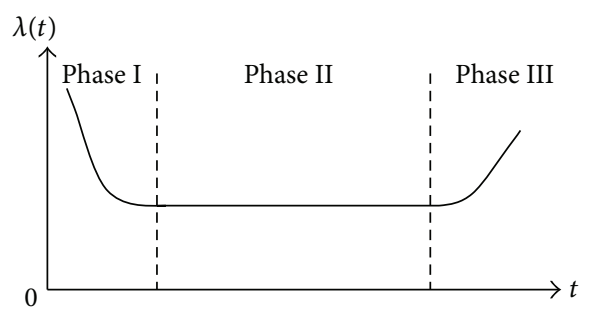

FIGURE 1

\section{Some Terminologies}

Some terms and their importance in this study are described below.

2.1. Reliability. It is a measure that the item/system will perform its required function under given conditions for stated time interval $[1,26,27]$ and it is denoted by $R(t)$. Mathematically

$$
\begin{aligned}
& R(t)=\operatorname{Pr}\{\text { no failure in }(0, t]\} \\
& \text { with } R(0)=1, \quad \lim _{t \rightarrow \infty} R(t)=0 .
\end{aligned}
$$

If the failure rate $\lambda(t)$ is constant, then $\lambda(t)=\lambda$ and $R(t)=\exp (-\lambda t)$. In many practical applications, failure rate of item/system is considered as constant by assuming the condition of as-good-as-new for every repaired item/system. It is well explained in [1] that the resultant bathtub curve as shown in Figure 1 of failure rate of large population of statistically identical and independent items gives three phases: (i) early failures, (ii) failures with constant failure rate, and (iii) wearout failures, in all the phases the period of the second one is very much useful for many real life situations.

2.2. Availability. It is the probability that a system will perform its required function at a given instant of time or over a stated period of time when operated and maintained in a prescribed manner $[1,26,27]$. The three classifications of availability over operational time are given as follows.

(i) Point availability: the probability of satisfactorily functioning of the system at a given instant of time $t$ is termed as point availability and denoted as $A(t)$.

(ii) Mission availability: it is the average availability over the interval $(0, T]$ and is expressed as

$$
A(T)=\left(\frac{1}{T}\right) \int_{0}^{T} A(t) d t
$$

(iii) Steady state availability: it is defined as the probability that the system is performing its intended function with maintenance strategy for long run time; that is, the point availability for steady state and is given as

$$
A=\lim _{t \rightarrow \infty} A(t) \text {. }
$$




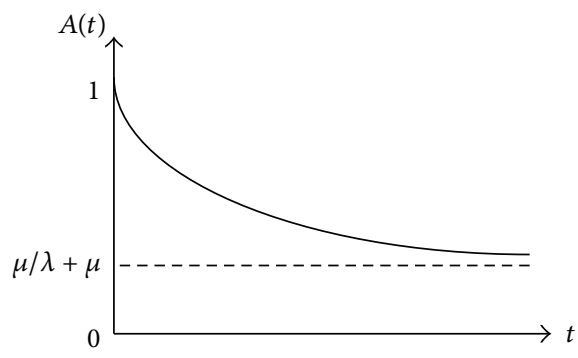

Figure 2

For single element system with constant failure and repair rates, availability is $A=\mu /(\lambda+\mu)$. Numerically, the difference between reliability and availability of the system is that for long run time reliability becomes zero, while availability goes to some fixed value as shown graphically in Figure 2.

2.3. Markov Process. Reliability and availability can easily be investigated in many industrial systems having structures convertible to reliability block diagram (RBD) but a tough situation arises when RBD of systems does not exist or cannot be easily found, as each component in RBD has only two states. To investigate these complex repairable systems various tools such as Markov process, semi-Markov, Petri nets, and dynamic FTA are developed. It is found from bathtub curve that failure and repair rates of components must be constant in real life situations and thus simplifying the system analysis. Hence system involving Markov process is considered in this discussion with certain assumptions. Markov process, a stochastic process exhibiting the memoryless property $[1,26$, 28 ] is a very powerful technique in the analysis of reliability and availability of complex repairable systems where the stay time in the system states follows an exponential distribution; that is, failure and repair rates are constant for all units during this process and the probability that the system changes its state depends only on the previous state. Finite state space and time-homogeneous Markov process is considered here and the diagram indicating the process is presented to develop differential equations.

2.4. Genetic Algorithm. A very powerful search technique in the field of optimization problems is genetic algorithm (GA) which has been initially introduced by Holland [29] and after that many researchers have carried their research with this technique [30-32]. Genetic algorithms are the combination of search algorithms which are based on the well-known biological fact of natural selection and natural genetics. These algorithms are very powerful for grasping improvement in their search and also computationally simple and thus they have wider area of applications such as business, scientific, and engineering. The main advantage of GA over other techniques is that it is not restricted by the condition on search spaces such as existence of continuity, differentiability, and unimodality. The flow chart depicting the process of GA is shown in Figure 3 and here the technique is executed by software MATLAB version 2008 with genetic algorithm and direct search toolbox.

\section{System Description}

Sugar mill, a very popular process industry that fulfils our various daily needs and other requirements, is considered here. Feeding, evaporation, and crystallization systems are the main constituents of sugar mill and their functioning/nonfunctioning affects the process of producing sugar from sugarcanes. Every subsystem of sugar industry has importance in its performance and hence each one plays a distinguished role in the overall system operation. In this section a subsystem of sugar industry, namely, feeding system for which the functioning or failure of any of its component affects the working process of the system in a certain manner, is described.

3.1. Feeding System Description. The feeding system of sugar industry has mainly four subsystems, namely, cutting system, crushing system, bagasse carrying system, and heat generating system [12] which are described as follows.

(1) Cutting system $(A)$ : a cutting system $(A)$ consists of units connected in series each one of which is a combination of a conveyor and a cutter; failure of any unit leads to the failure of the system. Sugarcane is cut into small pieces by the cutter for their use in the next stage.

(2) Crushing system (B): after cutting, sugarcane pieces are sent to crushing system where a combination of conveyor and crusher are connected in series to crush the pieces for the extraction of raw juice and failure of any one leads to the system failure.

(3) Bagasse carrying system $(C)$ : to use the crushed cane as a part of fuel for the boilers, there is a combination of carrier to carry the bagasse from crushing system to the heat generating system. The usage of bagasse may increase the efficiency of generating heat which is used for multiple purposes in the sugar system as for evaporation, crystallization, and so forth. The system $C$ has carriers connected in series and failure of anyone unit in $C$ reduces the efficiency of the system and also it affects the fuel supply to the next connected subsystem known as heat generating system $(D)$.

(4) Heat generating system $(D)$ : heat is very much required in the entire process of sugar mill and it is generated in the sugar mill itself by burning fuel such as coal, wood, and crushed cane (bagasse). Subsystem $D$ consists of parallel connected boilers in a way such that failure of any one component in $D$ reduces only the efficiency of the entire system.

\section{Assumptions and Notations}

(i) Failure-free time and repair time are stochastically independent and continuous random variables.

(ii) Failures are not considered during repair at system down state.

(iii) Repair is done according to first-in/first-out strategy and a repaired unit is assumed as-good-as-new. 


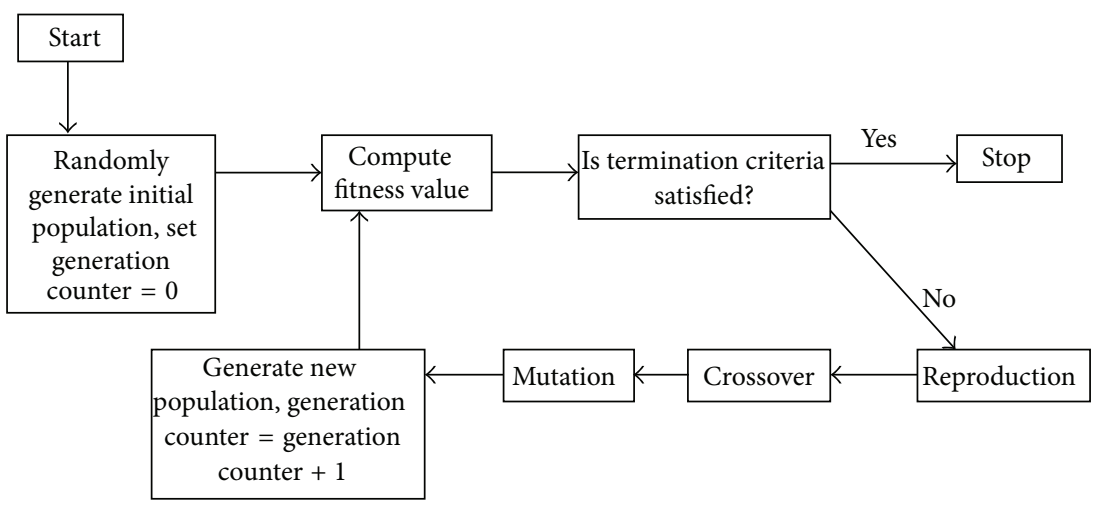

Figure 3

$A, B, C, D$ operative states of all four subsystems of feeding system.

$a, b, \bar{C}, \bar{D}$ failed states of systems $A, B$ and reduced state of $C, D$, respectively.

$\lambda_{A}, \lambda_{B}$ failure rates for complete failure of systems $A, B$.

$\mu_{A}, \mu_{B}$ repair rates of systems $A, B$.

$\alpha_{C}, \alpha_{D}, \beta_{C}, \beta_{D}$ transition rate of $C, D$ into $\bar{C}, \bar{D}$ and $\bar{C}, \bar{D}$ into $C, D$, respectively.

0 represents operative state.

$3,6,9$ represent reduced states, while $1,2,4,5$, $7,8,10$, and 11 represent failed states.

$p_{j}(t)$ represents the probability that the system is in state $j$ at time

$\bigcirc, \diamond, \square$ represent good, reduced, and failed states, respectively.

\section{Methodology}

It is clear from Section 2 and the literature that complex repairable systems must possess constant failure and repair rates for every unit in general. Thus the performance of a repairable system can be easily analysed by exhibiting timehomogeneous Markov process and modelling its finite states. By updating the Markov model, other useful measures are also easily obtained. The good decision of system parameters is always required for maximum performance and therefore for that an optimization technique is applied on the system availability model over a range of unknown variables. The main steps of the analysis done in this paper are pointed as follows:

(i) modelling the system states by Markov process, that is, taking constant failure and repair rates of each individual. Update the model by considering all failed states as absorbing states; that is, once entered in then it is impossible to leave that state;

(ii) obtain reliability function, steady state availability, and also state the optimization model; (iii) optimize the model by GA to obtain optimal design parameters and finally state all the values appropriate for system survivability by using an example.

5.1. Modelling by Markov Process. The system is modelled as in [12] but here we have considered that failure-free time as well as time involved in repair of failed components must be exponentially distributed. The corresponding transition diagram of involved states is shown in Figure 4.

To investigate Markov process, differential equations are easily derived from the transition diagram as

$$
\begin{aligned}
& \frac{d p_{0}(t)}{d t}+\left(\lambda_{A}+\lambda_{B}+\alpha_{C}+\alpha_{D}\right) p_{0}(t) \\
& =\mu_{A} p_{1}(t)+\mu_{B} p_{2}(t)+\beta_{C} p_{3}(t)+\beta_{D} p_{6}(t), \\
& \frac{d p_{3}(t)}{d t}+\left(\lambda_{A}+\lambda_{B}+\beta_{C}\right) p_{3}(t) \\
& \quad=\mu_{A} p_{4}(t)+\mu_{B} p_{5}(t)+\alpha_{C} p_{0}(t)+\beta_{D} p_{9}(t), \\
& \frac{d p_{6}(t)}{d t}+\left(\lambda_{A}+\lambda_{B}+\alpha_{C}+\beta_{D}\right) p_{6}(t) \\
& \quad=\mu_{A} p_{7}(t)+\mu_{B} p_{8}(t)+\alpha_{D} p_{0}(t), \\
& \frac{d p_{9}(t)}{d t}+\left(\lambda_{A}+\lambda_{B}+\beta_{D}\right) p_{9}(t) \\
& \quad=\mu_{A} p_{10}(t)+\mu_{B} p_{11}(t)+\alpha_{C} p_{6}(t), \\
& \frac{d p_{j}(t)}{d t}+\mu_{i} p_{j}(t)=\lambda_{i} p_{0}(t) \quad \text { where } \\
& (i, j) \text { belongs to }\{(A, 1)(B, 2)\},
\end{aligned}
$$

$\frac{d p_{j}(t)}{d t}+\mu_{i} p_{j}(t)=\lambda_{i} p_{3}(t) \quad$ where

$(i, j)$ belongs to $\{(A, 4)(B, 5)\}$,

$\frac{d p_{j}(t)}{d t}+\mu_{i} p_{j}(t)=\lambda_{i} p_{6}(t) \quad$ where

$(i, j)$ belongs to $\{(A, 7)(B, 8)\}$, 


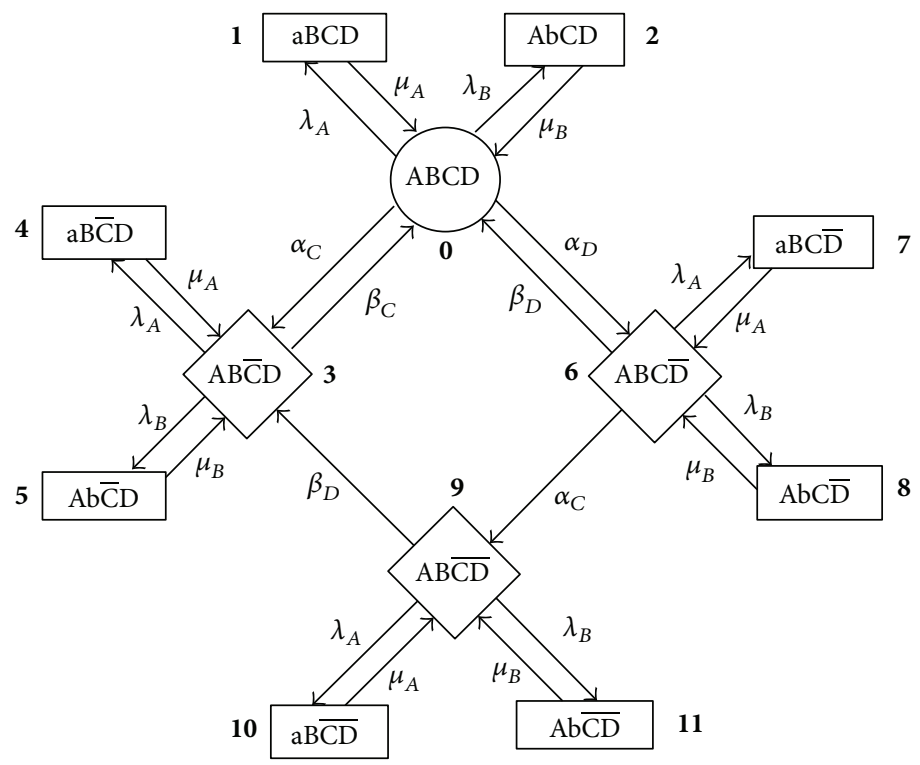

Figure 4

$\frac{d p_{j}(t)}{d t}+\mu_{i} p_{j}(t)=\lambda_{i} p_{9}(t) \quad$ where

$(i, j)$ belongs to $\{(A, 10)(B, 11)\}$.

A time-homogeneous Markov process is stationary if the state probabilities do not depend on time $t$ [1]. Therefore the steady state availability can be obtained by using the condition: as $t \rightarrow \infty, p j(t) \rightarrow p j$ and $d p j(t) / d t \rightarrow 0 \forall j$.

The above set of equations are then transformed into

$$
\begin{gathered}
\left(\lambda_{A}+\lambda_{B}+\alpha_{C}+\alpha_{D}\right) p_{0} \\
=\mu_{A} p_{1}+\mu_{B} p_{2}+\beta_{C} p_{3}+\beta_{D} p_{6}, \\
\left(\lambda_{A}+\lambda_{B}+\beta_{C}\right) p_{3} \\
=\mu_{A} p_{4}+\mu_{B} p_{5}+\alpha_{C} p_{0}+\beta_{D} p_{9}, \\
\left(\lambda_{A}+\lambda_{B}+\alpha_{C}+\beta_{D}\right) p_{6}=\mu_{A} p_{7}+\mu_{B} p_{8}+\alpha_{D} p_{0}, \\
\left(\lambda_{A}+\lambda_{B}+\beta_{D}\right) p_{9}=\mu_{A} p_{10}+\mu_{B} p_{11}+\alpha_{C} p_{6},
\end{gathered}
$$

$\mu_{i} p_{j}=\lambda_{i} p_{0}$ where $(i, j)$ belongs to $\{(A, 1)(B, 2)\}$,

$\mu_{i} p_{j}=\lambda_{i} p_{3}$ where $(i, j)$ belongs to $\{(A, 4)(B, 5)\}$,

$\mu_{i} p_{j}=\lambda_{i} p_{6}$ where $(i, j)$ belongs to $\{(A, 7)(B, 8)\}$,

$\mu_{i} p_{j}=\lambda_{i} p_{9} \quad$ where $(i, j)$ belongs to $\{(A, 10)(B, 11)\}$

Solving the system of equations recursively in terms of $p_{0}$, we get the equations as follows:

$$
p_{1}=\frac{\lambda_{A}}{\mu_{A}} p_{0}, \quad p_{2}=\frac{\lambda_{B}}{\mu_{B}} p_{0}, \quad p_{3}=\frac{\alpha_{C}\left(\alpha_{C}+\alpha_{D}+\beta_{D}\right)}{\beta_{C}\left(\alpha_{C}+\beta_{D}\right)} p_{0},
$$

$$
\begin{aligned}
& p_{4}=\frac{\lambda_{A} \alpha_{C}\left(\alpha_{C}+\alpha_{D}+\beta_{D}\right)}{\mu_{A} \beta_{C}\left(\alpha_{C}+\beta_{D}\right)} p_{0}, \\
& p_{5}=\frac{\lambda_{B} \alpha_{C}\left(\alpha_{C}+\alpha_{D}+\beta_{D}\right)}{\mu_{B} \beta_{C}\left(\alpha_{C}+\beta_{D}\right)} p_{0}, \\
& p_{6}=\frac{\alpha_{D}}{\left(\alpha_{C}+\beta_{D}\right)} p_{0}, \quad p_{7}=\frac{\lambda_{A} \alpha_{D}}{\mu_{A}\left(\alpha_{C}+\beta_{D}\right)} p_{0}, \\
& p_{8}=\frac{\lambda_{B} \alpha_{D}}{\mu_{B}\left(\alpha_{C}+\beta_{D}\right)} p_{0}, \quad p_{9}=\frac{\alpha_{C} \alpha_{D}}{\beta_{D}\left(\alpha_{C}+\beta_{D}\right)} p_{0}, \\
& p_{10}=\frac{\lambda_{A} \alpha_{C} \alpha_{D}}{\mu_{A} \beta_{D}\left(\alpha_{C}+\beta_{D}\right)} p_{0}, \quad p_{11}=\frac{\lambda_{B} \alpha_{C} \alpha_{D}}{\mu_{B} \beta_{D}\left(\alpha_{C}+\beta_{D}\right)} p_{0} .
\end{aligned}
$$

Now, using the normalizing condition

$$
\sum_{j=0}^{11} p_{j}=1
$$

in (6) we get,

$p_{0}$

$$
\begin{aligned}
= & {\left[1+\frac{\lambda_{A}}{\mu_{A}}+\frac{\lambda_{B}}{\mu_{B}}+\frac{\alpha_{C}\left(\alpha_{C}+\alpha_{D}+\beta_{D}\right)}{\beta_{C}\left(\alpha_{C}+\beta_{D}\right)}\right.} \\
& +\frac{\lambda_{A} \alpha_{C}\left(\alpha_{C}+\alpha_{D}+\beta_{D}\right)}{\mu_{A} \beta_{C}\left(\alpha_{C}+\beta_{D}\right)}+\frac{\lambda_{B} \alpha_{C}\left(\alpha_{C}+\alpha_{D}+\beta_{D}\right)}{\mu_{B} \beta_{C}\left(\alpha_{C}+\beta_{D}\right)} \\
& +\frac{\alpha_{D}}{\left(\alpha_{C}+\beta_{D}\right)}+\frac{\lambda_{A} \alpha_{D}}{\mu_{A}\left(\alpha_{C}+\beta_{D}\right)}+\frac{\lambda_{B} \alpha_{D}}{\mu_{B}\left(\alpha_{C}+\beta_{D}\right)} \\
& \left.+\frac{\alpha_{C} \alpha_{D}}{\beta_{D}\left(\alpha_{C}+\beta_{D}\right)}+\frac{\lambda_{A} \alpha_{C} \alpha_{D}}{\mu_{A} \beta_{D}\left(\alpha_{C}+\beta_{D}\right)}+\frac{\lambda_{B} \alpha_{C} \alpha_{D}}{\mu_{B} \beta_{D}\left(\alpha_{C}+\beta_{D}\right)}\right]^{-1} .
\end{aligned}
$$




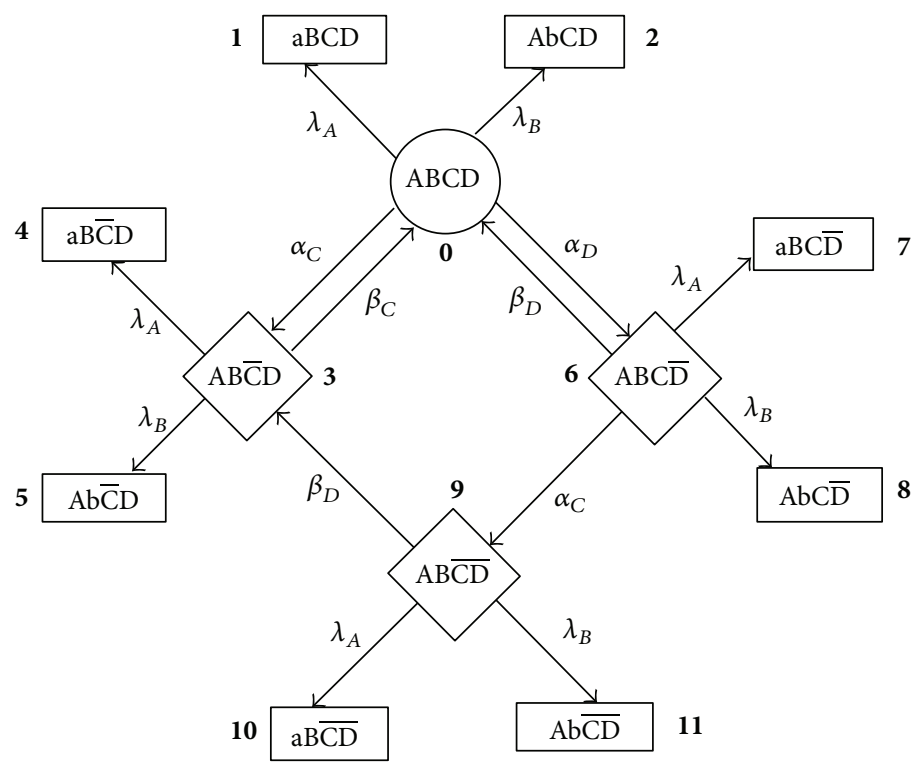

Figure 5

As the sum of state probabilities of all working states of the system leads to the system availability $[26,27]$, the steady state availability $A v_{S}$ is given by the equation as follows:

$A v_{S}$

$$
\begin{aligned}
& =p_{0}+p_{3}+p_{6}+p_{9} \\
& =\left[1+\frac{\alpha_{C}\left(\alpha_{C}+\alpha_{D}+\beta_{D}\right)}{\beta_{C}\left(\alpha_{C}+\beta_{D}\right)}+\frac{\alpha_{D}}{\left(\alpha_{C}+\beta_{D}\right)}+\frac{\alpha_{C} \alpha_{D}}{\beta_{D}\left(\alpha_{C}+\beta_{D}\right)}\right] p_{0} .
\end{aligned}
$$

In view of the value of $p_{0}$ as given by (8) the expression for steady state availability reduces to

$$
A v_{S}=\frac{1}{\left(1+\left(\lambda_{A} / \mu_{A}\right)+\left(\lambda_{B} / \mu_{B}\right)\right)} \approx 1-\left(\frac{\lambda_{A}}{\mu_{A}}+\frac{\lambda_{B}}{\mu_{B}}\right) \text {. }
$$

This expression is the same as for a two-element series system. Therefore cutting system $A$ and crushing system $B$ of sugar mill behave like a 2 -series system and hence the parameters related to $A$ and $B$ affect directly the productivity of the system.

To measure the reliability function associated with this Markov process, all the failed states in the transition diagram are considered as absorbing states [1] as shown in Figure 5.

The differential equations related to the transition diagram (Figure 5) are given as

$$
\begin{gathered}
\frac{d p_{0}(t)}{d t}+\left(\lambda_{A}+\lambda_{B}+\alpha_{C}+\alpha_{D}\right) p_{0}(t) \\
=\beta_{C} p_{3}(t)+\beta_{D} p_{6}(t), \\
\frac{d p_{3}(t)}{d t}+\left(\lambda_{A}+\lambda_{B}+\beta_{C}\right) p_{3}(t) \\
=\alpha_{C} p_{0}(t)+\beta_{D} p_{9}(t),
\end{gathered}
$$

$$
\begin{gathered}
\frac{d p_{6}(t)}{d t}+\left(\lambda_{A}+\lambda_{B}+\alpha_{C}+\beta_{D}\right) p_{6}(t)=\alpha_{D} p_{0}(t), \\
\frac{d p_{9}(t)}{d t}+\left(\lambda_{A}+\lambda_{B}+\beta_{D}\right) p_{9}(t)=\alpha_{C} p_{6}(t), \\
\frac{d p_{j}(t)}{d t}=\lambda_{i} p_{0}(t) \quad \text { where } \\
(i, j) \text { belongs to }\{(A, 1)(B, 2)\} \\
d p_{j}(t) / d t=\lambda_{i} p_{3}(t) \quad \text { where } \\
(i, j) \text { belongs to }\{(A, 4)(B, 5)\}, \\
d p_{j}(t) / d t=\lambda_{i} p_{6}(t) \quad \text { where } \\
(i, j) \text { belongs to }\{(A, 7)(B, 8)\}, \\
d p_{j}(t) / d t=\lambda_{i} p_{9}(t) \quad \text { where } \\
(i, j) \text { belongs to }\{(A, 10)(B, 11)\} .
\end{gathered}
$$

The above set of equations is a well-known system of linear differential equations with constant coefficients which is solved by various numerical methods. In this discussion, the system of equations is solved by eigenvalue method with $e_{i}$ as the eigenvalues and $v_{i}$ as their corresponding eigenvectors and the solution is expressed as

$$
\begin{gathered}
X(t)=\sum_{i=0}^{11} c_{i} \exp \left(e_{i} * t\right) v_{i} \quad \text { with } X(0)=\left[\begin{array}{llll}
1 & 0 & \cdots & 0
\end{array}\right]^{T}, \\
X(t)=\left[\begin{array}{llll}
p_{0}(t) & p_{1}(t) & \cdots & p_{11}(t)
\end{array}\right]^{T} .
\end{gathered}
$$

The reliability of system at any time $t$ is given as $R(t)=p_{0}(t)+$ $p_{3}(t)+p_{6}(t)+p_{9}(t)$ and mean time to failure of the whole 
system is MTTF $=\int_{t=0}^{\infty} R(t) d t$. Thus system involving Markov process is easily handled to find its reliability and availability by only modelling its corresponding transition diagrams for reliability function and availability function.

\section{Optimization Analysis}

In real life situations, system personnel is concerned about how to increase profit and productivity of the system and make the system highly reliable for long duration of time. Therefore, it is necessary while designing the system to know the information about its units which effect system's working capacity and also choose the best components which increase the overall functioning of the system for long run time with regard to their failures. Therefore our main purpose is to find the best system design units by selecting appropriate values of failure and repair rates within the specified ranges such that the system long run availability maximizes. The ranges or crisp values of failure and repair rates of the system can be found by reviewing the previous records of that system or by experimental process or by concerning that particular system analyst.

In this study, to analyse and measure the performance factors, we consider a physical situation where each subsystem of feeding system has a mean failure rate of 0.001 to 0.002 per operating hour and mean repair rate of 0.2 to 0.4 per operating hour and the system is configured as $A$ and $B$ having four components each, $C$ having three components, and $D$ having six components. Now for each macrostructure, their individual failure rate and repair rate are calculated by the formula given as follows:

$$
\begin{gathered}
\lambda_{A}=\sum_{i=1}^{4} \lambda_{i}, \quad \mu_{A} \approx \frac{\lambda_{1}+\lambda_{2}+\cdots+\lambda_{4}}{\left(\lambda_{1} / \mu_{1}\right)+\left(\lambda_{2} / \mu_{2}\right)+\cdots+\left(\lambda_{4} / \mu_{4}\right)}, \\
\lambda_{B}=\sum_{i=1}^{4} \lambda_{i}, \quad \mu_{B} \approx \frac{\lambda_{1}+\lambda_{2}+\cdots+\lambda_{4}}{\left(\lambda_{1} / \mu_{1}\right)+\left(\lambda_{2} / \mu_{2}\right)+\cdots+\left(\lambda_{4} / \mu_{4}\right)}, \\
\alpha_{C}=\sum_{i=1}^{3} \alpha_{i}, \quad \beta_{C} \approx \frac{\alpha_{1}+\alpha_{2}+\alpha_{3}}{\left(\alpha_{1} / \beta_{1}\right)+\left(\alpha_{2} / \beta_{2}\right)+\left(\alpha_{3} / \beta_{3}\right)}, \\
\alpha_{D}=\sum_{i=1}^{6} \alpha_{i}, \quad \beta_{D} \approx \frac{\alpha_{1}+\alpha_{2}+\cdots+\alpha_{6}}{\left(\alpha_{1} / \beta_{1}\right)+\left(\alpha_{2} / \beta_{2}\right)+\cdots+\left(\alpha_{6} / \beta_{6}\right)} .
\end{gathered}
$$

Hence the steady state availability optimization model becomes

$$
\begin{aligned}
\text { Maximize } & A v_{S}=\left[1+\frac{\lambda_{A}}{\mu_{A}}+\frac{\lambda_{B}}{\mu_{B}}\right]^{-1} \\
\text { s.t. } & 0.004 \leq \lambda_{A} \leq 0.008, \quad 0.2 \leq \mu_{A} \leq 0.4 \\
& 0.004 \leq \lambda_{B} \leq 0.008, \quad 0.2 \leq \mu_{B} \leq 0.4 .
\end{aligned}
$$

By taking the fitness function as the objective function in minimization form, the optimal values of failure and repair

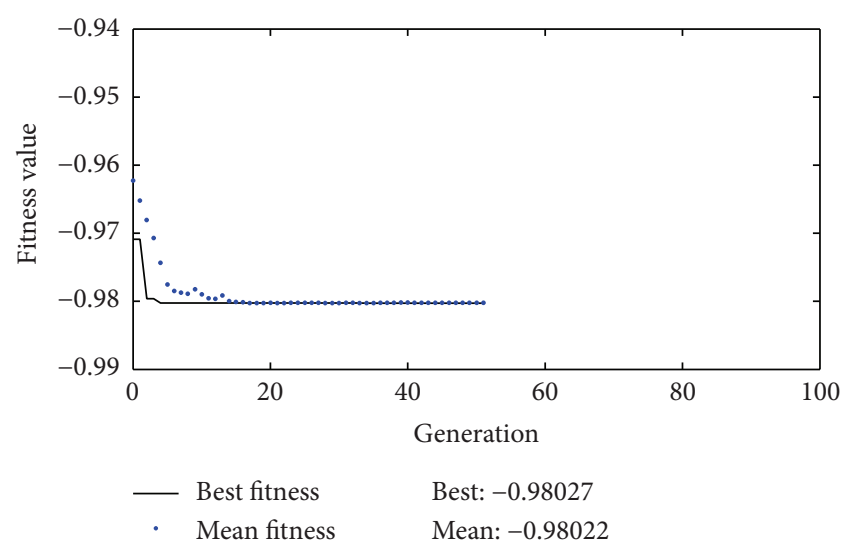

Figure 6

TABLE 1: Optimal design parameters.

\begin{tabular}{lcc}
\hline Components & Failure rate & Repair rate \\
\hline Cutting system & 0.004 & 0.4 \\
Crushing system & 0.004 & 0.395 \\
Bagasse carrying system & 0.0045 & 0.3 \\
Heat generating system & 0.009 & 0.3 \\
\hline
\end{tabular}

rates are calculated by executing genetic algorithm with the help of MATLAB genetic algorithm and direct search tool box with installed settings. The best individual is taken by executing 20 runs and its performance graph with the generation count is shown in Figure 6. It is found that the technique approaches to the nearly optimal solution from generation count 20 with a very minor difference between the best and mean of fitness value. Thus the best system availability and the optimal parameters after this execution come out to be 0.980274 , and $\lambda_{A}=0.004, \mu_{A}=0.4, \lambda_{B}=$ $0.004, \mu_{B}=0.395$ within 51 generation counts, respectively.

From above analysis it is clear that the failure and repair rates of subsystems $C$ and $D$ do not affect the working capacity of system for long time period. For evaluation of reliability at a time, it is best to choose the corresponding data as their average values as there is no significant variation in the system reliability with respect to their rates. Therefore the optimal design policy resulting system availability as 0.980274 is stated in Table 1 . For this strategy, the system reliability at different time stages within one hour interval is found by using (12) and coding it into MATLAB with the condition of ignoring the imaginary part of any numerical value and is stated in Table 2 for initial hours. The reliability curve up to 1000 hours for optimal design policy is shown in Figure 7. Thus we conclude that various performance measures of the systems are easily obtained by considering time-homogeneous Markov process and after that according to them the impact of each subsystem can be found.

\section{Discussion}

In the present era of technology, industrial systems are structured with more standby units/subsystems in a particular 
TABLE 2: System reliability after $t$-hrs.

\begin{tabular}{|c|c|c|c|c|c|c|c|c|}
\hline$t$ (hrs) & 0 & 10 & 50 & 100 & 150 & 200 & 250 & 500 \\
\hline$R(t)$ & 1 & 0.924289 & 0.679017 & 0.461945 & 0.314268 & 0.213801 & 0.145452 & 0.021197 \\
\hline
\end{tabular}

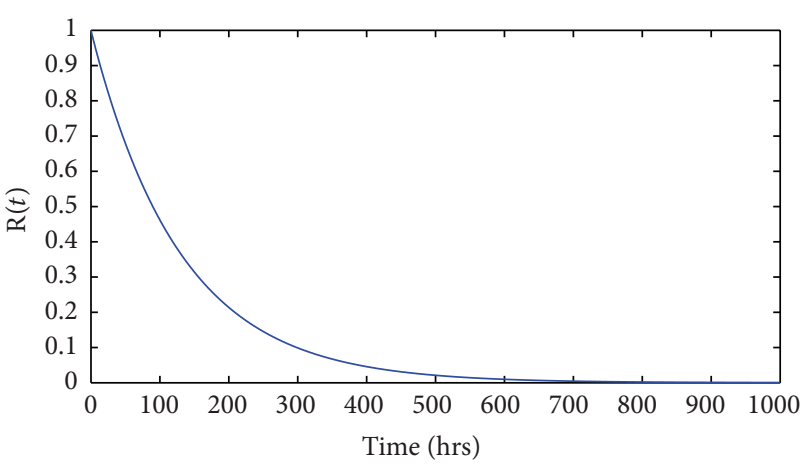

FIGURE 7: Reliability curve.

manner to increase the efficiency and duration of their operation. Thus complexity in system's structure arises as it exhibits more than two states and cannot be represented by reliability block diagram. In industries, performance analyses are necessary to make them more reliable by demonstrating their operational behaviour. To analyse the reliability and availability of feeding system of sugar industry, Markovian approach with genetic algorithm has been considered in this study. From the analysis, it is found that both performance measures of a complex system can easily be obtained by modelling the system states as Markovian approach with and without absorbing states. Apart from this approach, techniques such as dynamic Petri nets, dynamic fault tree analysis, and other computer based techniques are available in the literature [1]. Generally, the Petri nets are same as Markov model but its modelling process is quite tedious. Its graph includes places, transitions, and directed arcs with well-defined rules for transition of tokens, placed inside the places. Dynamic Petri nets are based on the criteria of firing of tokens, marking, reachability tree, and so forth, and it is necessary for system analyst having the knowledge of these important terms. More detailed explanation about Petri nets is given in $[1,33]$. In the case of complex system as taken in this paper it is found that Markov model can be formed in a simple and easy way by considering certain assumptions and the system reliability and availability can be analysed without any computational difficulty. Dynamic Petri nets also provide system performance measures but in a lengthy way. Another famous technique, dynamic fault tree analysis (dynamic FTA) is a graphical representation of conditions causing the occurrence of an undesirable event with dynamic gates [1]. FTA is used in variety of reliability oriented problems and involves fault trees, reliability block diagrams, and binary decision diagrams whereas dynamic FTA also requires the knowledge of various dynamic gates as PAND, SEQ, SPARE, and so forth [1]. Therefore Markov approach is found to be a simple and effective investigation tool in comparison to other techniques for the analysis of complex systems exhibiting more than two states.

\section{Conclusion}

In this paper, the application of time-homogeneous Markov process is explained in the operating process of feeding system of a well-popular sugar industry. It is clearly shown that the expression for reliability function and availability for a complex repairable system having more than two states is easily obtained by system Markov modelling and this method is found very useful in calculating these measures for any industrial systems. To design the feeding system in a way such that it will survive for long time period, we have assumed the data regarding to its configuration and shown the proper use of Markov process and a search technique Genetic Algorithm. To run GA with more efficiency, popular MATLAB software with genetic algorithm optimization tool is executed. This study states that a system can be analysed easily by concerning the process as Markov process and it helps the system design analyst or plant personnel to select the most appropriate structural components. Thus this discussion assists the system in achieving its high performance measures for maximum duration of time.

\section{Conflict of Interests}

The authors declare that there is no conflict of interests regarding the publication of this paper.

\section{Acknowledgment}

The corresponding author would like to thank the Ministry of Human Resource and Development, New Delhi, India, for providing financial support during the research work.

\section{References}

[1] A. Birolini, Reliability Engineering: Theory and Practice, Springer, New York, NY, USA, 5th edition, 2007.

[2] W. Kuo, V. R. Prasad, F. A. Tillman, and C. L. Hwang, Optimal Reliability Design-Fundamentals and Applications, Cambridge University Press, Cambridge, UK, 2001.

[3] A. Adamyan and D. He, "System failure analysis through counters of Petri net models," Quality and Reliability Engineering International, vol. 20, no. 4, pp. 317-335, 2004.

[4] S. Aksu, S. Aksu, and O. Turan, "Reliability and availability of pod propulsion systems," Quality and Reliability Engineering International, vol. 22, no. 1, pp. 41-58, 2006.

[5] J. Knezevic and E. R. Odoom, "Reliability modelling of repairable systems using Petri nets and fuzzy Lambda-Tau 
methodology," Reliability Engineering and System Safety, vol. 73, no. 1, pp. 1-17, 2001.

[6] Komal, S. P. Sharma, and D. Kumar, "RAM analysis of the press unit in a paper plant using genetic algorithm and lambda-tau methodology," in Proceeding of the 13th Online International Conference (WSC '08), vol. 58 of Applications of Soft computing (Springer Book Series), pp. 127-137, 2009.

[7] R. K. Sharma and S. Kumar, "Performance modeling in critical engineering systems using RAM analysis," Reliability Engineering and System Safety, vol. 93, no. 6, pp. 913-919, 2008.

[8] B. S. Dhillion and C. Singh, Engineering Reliability: New Techniques and Applications, John Wiley \& Sons, New York, NY, USA, 1991.

[9] S. P. Sharma and H. Garg, "Behavioural analysis of urea decomposition system in a fertiliser plant," International Journal of Industrial and Systems Engineering, vol. 8, no. 3, pp. 271-297, 2011.

[10] H. Garg and S. P. Sharma, "Behavior analysis of synthesis unit in fertilizer plant," International Journal of Quality and Reliability Management, vol. 29, no. 2, pp. 217-232, 2012.

[11] D. Kumar, J. Singh, and P. C. Pandey, "Availability of a washing system in the paper industry," Microelectronics Reliability, vol. 29, no. 5, pp. 775-778, 1989.

[12] D. Kumar, J. Singh, and I. P. Singh, "Availability of the feeding system in the sugar industry," Microelectronics Reliability, vol. 28, no. 6, pp. 867-871, 1988

[13] N. Arora and D. Kumar, "Availability analysis of steam and power generation systems in the thermal power plant," Microelectronics Reliability, vol. 37, no. 5, pp. 795-799, 1997.

[14] P. Gupta, "Markov Modeling and availability analysis of a chemical production system-a case study," in Proceedings of the World Congress on Engineering (WCE '11), pp. 605-610, London, UK, July 2011.

[15] S. Kumar and P. C. Tewari, "Mathematical modelling and performance optimization of $\mathrm{CO}_{2}$ cooling system of a Fertilizer plant," International Journal of Industrial Engineering Computations, vol. 2, no. 3, pp. 689-698, 2011.

[16] P. Gupta, A. K. Lal, R. K. Sharma, and J. Singh, "Numerical analysis of reliability and availability of the serial processes in butter-oil processing plant," International Journal of Quality and Reliability Management, vol. 22, no. 3, pp. 303-316, 2005.

[17] R. Kumar, A. K. Sharma, and P. C. Tewari, "Markov approach to evaluate the availability simulation model for power generation system in a thermal power plant," International Journal of Industrial Engineering Computations, vol. 3, no. 5, pp. 743-750, 2012.

[18] M. Manglik and M. Ram, "Reliability analysis of a two unit cold standby system using Markov process," Journal of Reliability and Statistical Studies, vol. 6, no. 2, pp. 65-80, 2013.

[19] R. Kumar, "Availability analysis of thermal power plant boiler air circulation system using Markov approach," Decision Science Letters, vol. 3, no. 1, pp. 65-72, 2014.

[20] F. A. Tillman, C. L. Hwang, and W. Kuo, Optimization of Systems Reliability, Marcel Dekker, New York, NY, USA, 1980.

[21] V. Ravi, B. S. N. Murty, and P. J. Reddy, "Nonequilibrium simulated annealing-algorithm applied to reliability optimization of complex systems," IEEE Transactions on Reliability, vol. 46, no. 2, pp. 233-239, 1997.

[22] V. Ravi, P. J. Reddy, and H.-J. Zimmermann, "Fuzzy global optimization of complex system reliability," IEEE Transactions on Fuzzy Systems, vol. 8, no. 3, pp. 241-248, 2000.
[23] H. Garg, M. Rani, and S. P. Sharma, "An approach for analyzing the reliability of industrial systems using soft-computing based technique expert systems with applications," vol. 41, no. 2, pp. 489-501, 2014.

[24] R. Khanduja, P. C. Tewari, R. S. Chauhan, and D. Kumar, "Mathematical modelling and performance optimization for the paper making system of a paper plant," Jordan Journal of Mechanical and Industrial Engineering, vol. 4, no. 4, pp. 487494, 2010.

[25] S. P. Sharma, D. Kumar, and A. Kumar, "Availability optimization of a series-parallel systems using genetic algorithms," in Proceedings of the 32nd National Systems Conference (NSC '08), December 2008.

[26] C. E. Ebeling, An Introduction to Reliability and Maintainability Engineering, McGraw-Hill, New York, NY, USA.

[27] L. S. Srinath, Reliability Engineering, East-West Press Private Limited, New Delhi, India, 4th edition, 2005.

[28] J. K. Kitchin, "Practical Markov modelling for reliability analysis," in Proceedings of the Annual Reliability And Maintainability Symposium, pp. 290-296, Los Angeles, Calif, USA, 1988.

[29] J. H. Holland, Adaptation in Natural and Artificial Systems, The University of Michigan Press, Ann Arbor, Mich, USA, 1975.

[30] D. E. Goldberg, Genetic Algorithms in Search, Optimization and Machine Learning, Addison-Wesley, New York, NY, USA, 1989.

[31] K. Deb, "Optimal design of a welded beam structure via genetic algorithms," AIAA Journal, vol. 29, no. 11, pp. 2013-2015, 1991.

[32] S. Rajeev and C. S. Krishnamoorthy, "Discrete optimization of structures using genetic algorithms," Journal of Structural Engineering, vol. 118, no. 5, pp. 1233-1250, 1992.

[33] W. Reisig, Petri Nets: An Introduction, Springer, Berlin, Germany, 1985. 


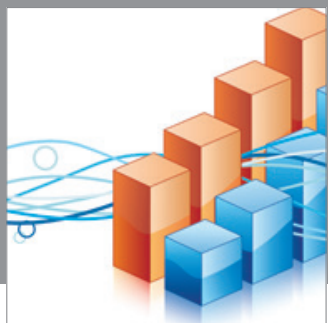

Advances in

Operations Research

mansans

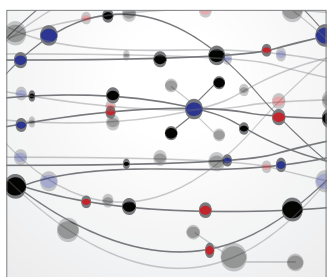

The Scientific World Journal
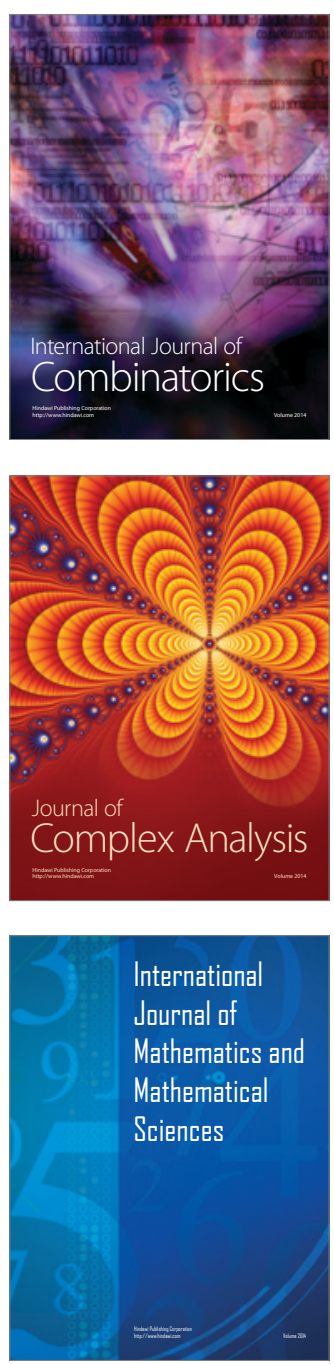
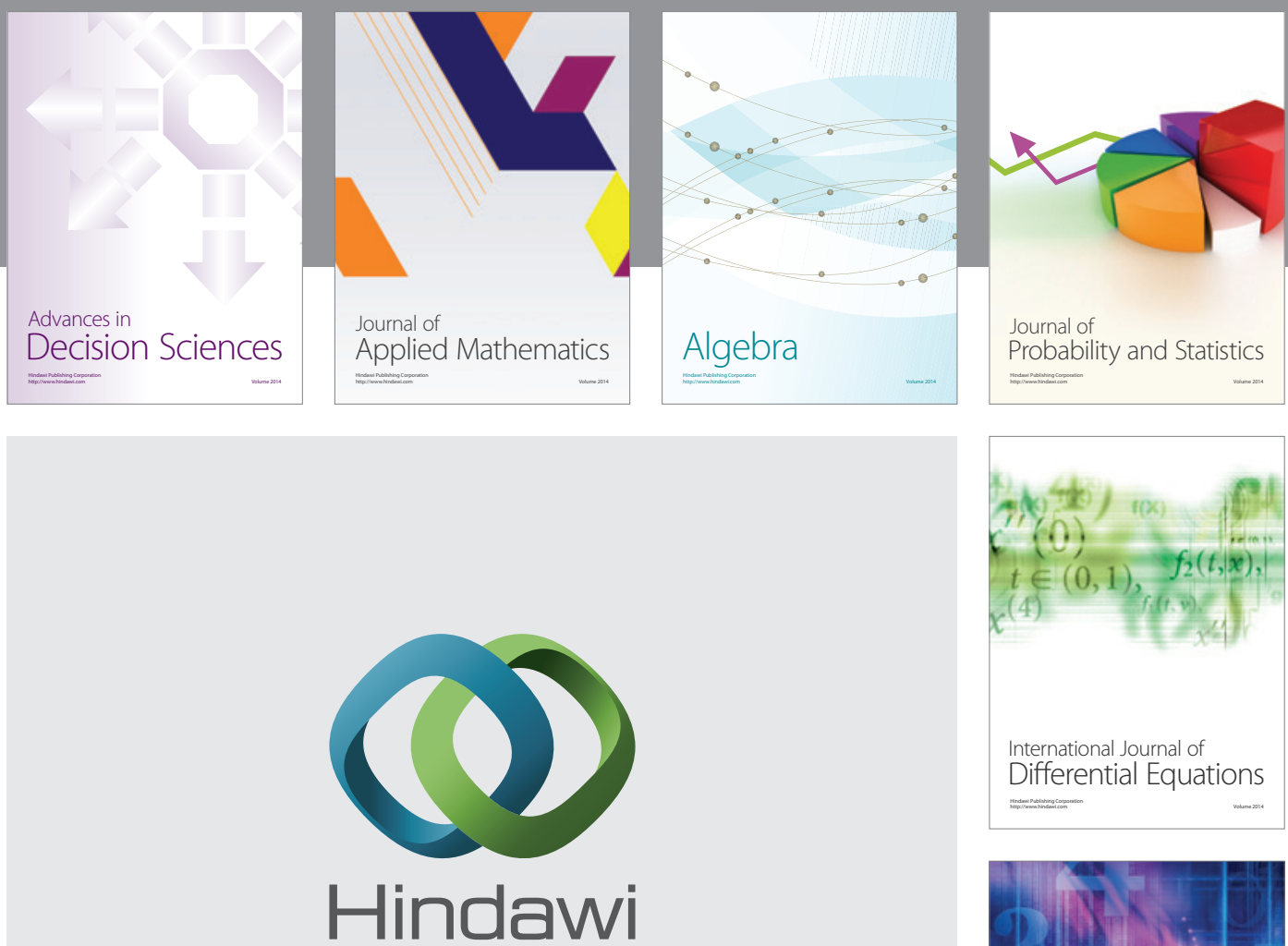

Submit your manuscripts at http://www.hindawi.com
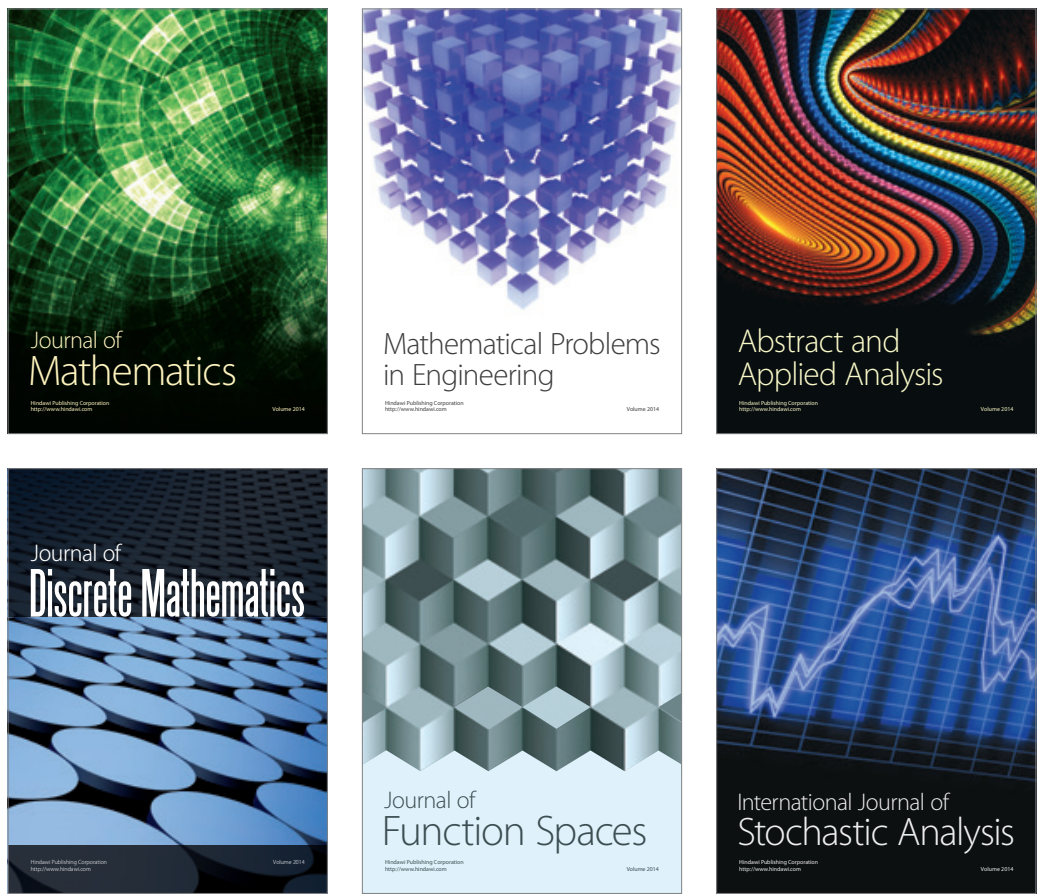

Journal of

Function Spaces

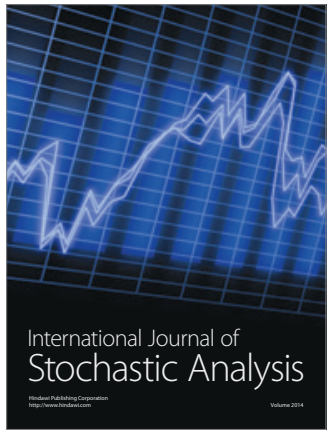

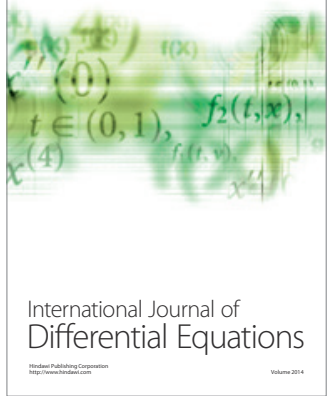
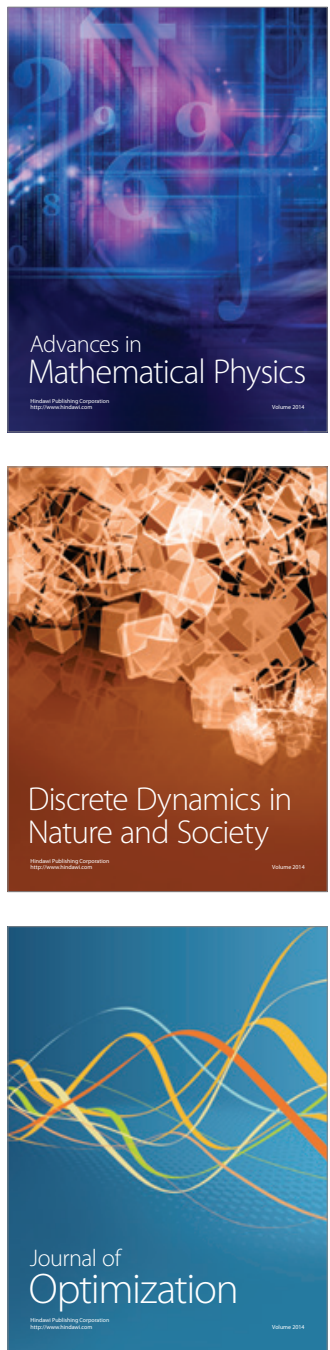